\title{
Current Drive operation in a In-blanket ICRF Ring Array
}

\author{
G.Bosia $^{1 *}$, R. Ragona ${ }^{2}$ \\ ${ }^{1}$ Dept of Physics, University of Turin and INFN, Via P. Giuria 110125 Turin ${ }^{*}$ \\ ${ }^{2}$ Ghent University, 9000 Ghent, Belgium
}

\begin{abstract}
The currently predicted Current Drive (CD)efficiency in a tokamak reactor plasma is $\chi_{C D} \leq 50 \mathrm{kA} / \mathrm{MW}$ for all auxiliary systems (NBCD, ICCD, ECCD). This would imply a relatively low reactor $Q=P_{\text {electric }} / P_{\text {auxiliary }}$ and an extremely high power density $\left(>10 \mathrm{MW} / \mathrm{m}^{2}\right)$ at the plasma boundary, if injected through the vacuum vessel ports or by in-port launchers..

In a previous paper the concept of an Ion Cyclotron Ring Array (RA) of many elements, integrated in the reactor blanket first wall was proposed. An analysis of this RA operating in steady state Fast Wave Current Drive (FW CD) with all elements individually controlled in magnitude and phase is presented in this paper. For CD operation the RA is operated at low frequency ( $\mathrm{f} \approx 20 \mathrm{MHz}$ ) so as to avoid the otherwise overwhelming RF power absorption by ions and $\alpha$-particles. It is shown that the RA would feature a very directive radiation spectrum, a low power density operation (avoiding the drawbacks associated with high AC and DC potentials) and a potentially high flexibility of operation..
\end{abstract}

\section{Introduction}

Current drive (CD) generation in a tokamak is the result of a rather complex physics process requiring a toroidal momentum transfer (by ELD + TTMP) to the plasma electrons travelling at an average toroidal thermal speed resonant with the toroidal phase velocity of a FW injected at the plasma boundary. The competition of this process with the more efficient power absorption by ions or fusion-born $\alpha$-particles significantly impairs the CD efficiency and needs to be avoided either by using specific frequency windows where ion absorption is minimized or, alternatively, by operation at a frequency sufficiently low to keep ion resonances outside the plasma cross section, as proposed here.

Steady state operation is regarded to be essential for a future commercial reactor, but the predicted CD efficiency of the "injected" auxiliary systems (NBCD and ECCD) is rather low $\left(\chi_{C D} \leq 0.05 A / W\right)$ even in a high $T_{e}$ reactor [1], requiring, among other problems, an extremely high power density at the plasma edge and the use of large apertures in the vacuum vessel and blanket for direct plasma access. An ICRF launcher, necessarily located inside the vessel because of the required proximity to the plasma edge imposed by the wave evanescence, would suffer a similar power density problem, if restricted in a port. If instead a wider surface of the blanket first wall could be used, it could operate at a significantly lower AC and DC electric fields and therefore avoid the drawbacks associated with the FW evanescence (voltage breakdowns and impurity influxes due to rectified sheath potentials).

The FW CD efficiency computed for a launching structure similar to the (four straps) ITER launcher installed in DEMO (commercial fusion reactor conceptual study [2]) by using the Ehst \& Karney approximate formula [3] is predicted at values close to those of the other auxiliary systems $[4,5]$. It is found that the CD efficiency depends on the so called E\&K parameter $w=v_{p h} / v_{e}$, increasing for $w>1$ and sharply decreasing for $w<1$ as in this range the RF power is preferentially coupled to off-axis, magnetically trapped electrons, not contributing to CD.
On the other hand, the Single Pass Absorbed (SPA) power by electrons is expected to vary as $p_{e} \approx w e^{-w^{2} / 2}$ [6], i.e. to rapidly decrease for $\mathrm{w}>1$. All studies devoted to DEMO steady state operation use the ITER in-port array (defined by its toroidal mode number $n_{\varphi} \approx 45$ ) as reference. It is shown in [7] that the competition with ions and fusion born $\alpha$-particles absorption, would restrict FWCD to relatively narrow high frequency windows (e.g. at $\mathrm{f} \approx 51 \mathrm{MHz}$ ). The best predicted $\mathrm{CD}$ efficiency for an equatorial $\mathrm{FW}$ launch is $\eta_{C D}=0.03 \mathrm{~A} / \mathrm{W}$, whereas in [8] it is shown that for operation at low frequency ( $\mathrm{f}<20 \mathrm{MHz}$ in DEMO, avoiding ion absorption competition), the $\mathrm{CD}$ efficiency of an ITER-like antenna would reduce to $\approx 0.012 \mathrm{~A} / \mathrm{W}$, due to a high off-axis magnetic trapping (against a value of $\approx 0.086 .1 \mathrm{~A} / \mathrm{W}$ in absence of trapping).

In [9] an all-metal Ion Cyclotron Ring Array (RA), in principle compatible with reactor operation, is proposed for heating and CD use in DEMO.

In this paper the $\mathrm{CD}$ performance of a toroidally resonant RA launcher radiating from the low field side are examined in a DEMO-like reactor and approximate relations useful as a guide of a realistic RA design are deduced.

\section{Ring Array operation}

The RA is a toroidal periodic structure of $N_{a}$ poloidal strip line elements with poloidal length $l_{y} \ll \lambda_{y},\left(\lambda_{y}\right.$ designed to be integrated in the first wall of the blanket of a tokamak fusion reactor. The strip lines currents $\mathrm{I}_{0}$ (or the input voltages $\mathrm{V}_{0}$ ) are individually controlled at an equal magnitude and toroidal phase advances $\theta_{\varphi_{r}}\left(r=1, . . N_{a}\right)$ by balanced feedback-controlled current sources (Fig.1).

The RA is designed to be integrated in the first wall of the blanket of a tokamak fusion reactor. All elements are mechanically supported at mid length to the back plate or, if present, to a slotted Faraday shield (both at ground potential). and fed at both ends The array of short strip line elements $\left(l_{y} \ll \lambda_{y}\right)$ is designed to operate at a power level of several tens of MW and features a small radial depth $(15-20 \mathrm{~cm})$, for minimum impact with the blanket functions. As radiation losses to

\footnotetext{
Corresponding author: gbosia@,to.infn.it
} 
the plasma are periodically restored by the current sources, the RA may be regarded as an overall lossless toroidal periodic structure. According to the Floquet theorem, the field components of the structure can be expanded in spatial harmonics in the form:

$$
\psi(R, \varphi)=\sum_{n=-\infty}^{\infty} \psi_{n}(R) e^{\left.-i\left(\beta+n N_{a}\right)\right) \varphi}
$$

where $\beta=\omega / v_{p h_{\phi}}$ is the propagation constant and $v_{p h_{\phi}}$ is the toroidal phase velocity. By setting the toroidal boundary conditions it can be shown that the array toroidal mode number $n_{\varphi}$, the toroidal phase velocity and the operating frequency are related to the number of elements $N_{a}$ and to phase advance $\theta_{\varphi}$, as listed in Table 1

Table 1.

\begin{tabular}{|l|l|}
\hline Toroida.mode number & $n_{\varphi}=N_{a} \frac{-\theta_{\varphi}}{2 \pi}$ \\
\hline Electrons therm. velocity & $v_{\text {th.e }}=c \sqrt{T_{e} / m_{e} c^{2}}$ \\
\hline Toroidal. phase velocity & $v_{p h . \varphi}=\frac{\omega R_{a}}{n_{\varphi}}$ \\
\hline E \&K parameter & $w=\frac{R_{a} \omega}{n_{\varphi} c} \frac{-\theta_{\varphi}}{\pi} \sqrt{m_{e} c^{2} / T_{e}}$ \\
\hline frequency & $f=w n_{\varphi} \sqrt{T_{e} / m_{e} c^{2}}\left(c / 2 \pi R_{a}\right)$ \\
\hline
\end{tabular}

Due to the limitations in space of this paper the reader is referred to [11] or to the accompanying poster for more details on this subject.

\section{Ring Array Power Spectrum}

The current density per unit length (p.u.l.) $J_{y}(\varphi)$ $(\mathrm{A} / \mathrm{m})$ of a ring structure with elements located at angles $\varphi_{n}$ can be written as:

$$
J_{y}(\varphi)=J_{o} \sum_{n=1}^{N_{a}} e^{i \varphi_{n}} \Theta_{\mathrm{n}}(\varphi) \quad(\mathrm{A} / \mathrm{m})
$$

with a rectangular function for the $\varphi$ dependance

$\Theta_{\mathrm{n}}(\varphi)=$

$e^{-i(n-1) \theta_{\varphi}}\left\{\begin{array}{c}1, \text { if }(n-1) p_{\varphi}<\varphi<(n-1) \\ p_{\varphi}+w_{\varphi}<0 \\ 0, \text { otherwise }\end{array}\right.$

where $p_{\varphi}=\frac{2 \pi}{N_{a}}$ is the array period, $w_{\varphi}=\xi p_{\varphi}$. is the stripline angular width such that $I_{0}=\int_{-w_{\varphi} / 2}^{w_{\varphi} / 2} J_{0 . y}(\varphi) d \varphi$ $=J_{0} l_{y} w_{\varphi}$. If a phase advance $\varphi_{n}=n \theta_{\varphi}$ is assumed

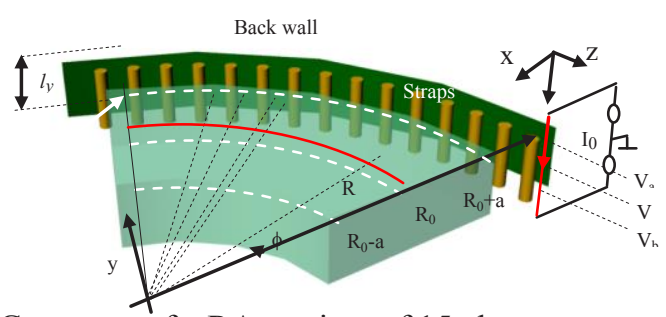

Fig 1. Geometry of a RA section of 15 elements

the Fourier transform of $J_{y}(\varphi)$ is:

$J_{y}\left(k_{\varphi}, p_{\varphi}, \theta_{\varphi}\right)=\frac{J_{0}}{\sqrt{2 \pi}} w_{\varphi} e^{-i, \frac{k_{\varphi} w_{\varphi}}{2}} \frac{\sin \left(\frac{k_{\varphi} w_{\varphi}}{2}\right)}{\frac{k_{\varphi} w_{\varphi}}{2}} S\left(k_{\varphi}\right)$ with $k_{\varphi}=\sum_{n=0}^{N_{a}-1} e^{-i n\left(k_{\varphi} p_{\varphi}+\theta_{\varphi}\right)}$. The active power $\left.P_{a}\left(k_{\varphi}, p_{\varphi}\right) \propto \int_{-\infty}^{+\infty} J_{y}\left(k_{\varphi},\right)\right|^{2} \mathrm{~d} k_{\varphi} \quad$ coupled to the plasma is proportional to $\left|S\left(k_{\varphi}\right)\right|^{2}$ which depends on $n_{\varphi}$ and $\theta_{\varphi}$. For $N_{a}>>1$ the power spectrum consists of sharply peaked distributions, with the main peak of emission at $k_{\varphi . m}=\frac{-\theta_{\varphi}}{p_{\varphi}}$ and width $\Delta k_{\varphi} \approx-\theta_{\varphi}$. In the asymptotic case $N_{a} \rightarrow \infty, \quad\left|J_{y}\left(k_{\varphi}, \pi / 2\right)\right| \rightarrow \infty$ and $\Delta k_{\varphi} \rightarrow 0$. Therefore, the spectral power distribution tends to $\delta\left(k_{\varphi}-n_{\varphi . m}\right)$. Plots of $\left|J_{y}\left(k_{\varphi}, \Delta \theta\right)\right|^{2}$ for a RA of radius $\mathrm{R}_{\mathrm{a}}=11.32 \mathrm{~m}$ with phase advance $\theta_{\varphi}=$ $\pi / 2, \mathrm{f}=20 \mathrm{MHz}$ and $n_{\varphi}==5,10,15,20,45$ are shown in Fig. 2 as function of $k_{\varphi}$.

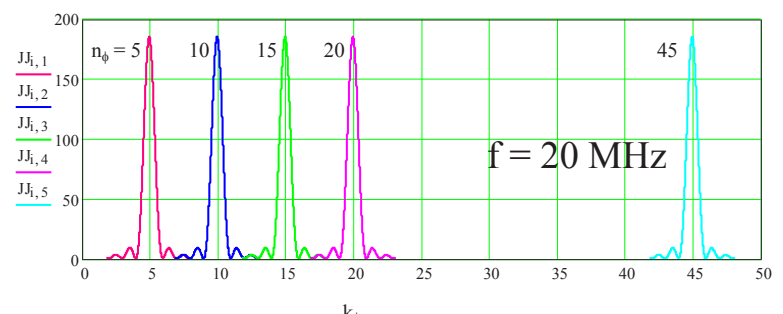

Fig 2. Power spectra vs. increasing values of $n_{\varphi}$

\section{Plasma loading}

The amount of power tunnelling through the evanescence layer is estimated by a $2 \mathrm{D}$ model of antenna coupling theory in quasi static approximation $\left(2 \pi / \mathrm{k}_{0} \gg \mathrm{a}, \mathrm{b}, \mathrm{d}, \mathrm{w}_{\mathrm{z}}\right)$ as function of $k_{z}=k_{\varphi} / R_{a}$, (the same symbols as in the original paper are used). Invariance with y $\left(\frac{\partial}{\partial y}=0\right)$ and a rectangular current density profile vs. $\varphi$ is assumed for the array elements. Therefore, all electrical quantities are defined per unit length (p.u.1). The effects of feeders and inter element coupling (which in turn depend on plasma coupling) are ignored. This implies an array geometry of loosely coupled elements. For $l_{y} \ll \lambda_{y}$, the strip lines operate close to anti-resonance. Therefore, the effects of the distributed capacitances are small and will be neglected. For the same reason, the assumption of a constant inductive strip line reactance p.u.l. is assumed. Using the definitions adopted in [10], the complex plasma loading impedance p.u.l of a single element is $Z_{y}=Z_{A} / N_{a}=R_{y}+i X_{y}$, with:

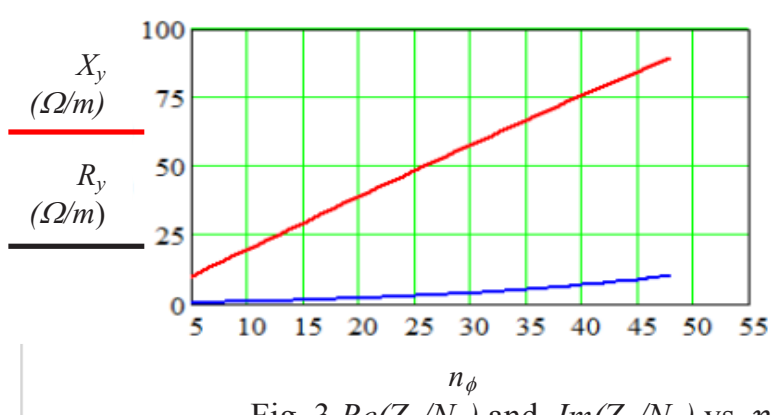

Fig. $3 \operatorname{Re}\left(Z_{A} / N_{A}\right)$ and $\operatorname{Im}\left(Z_{A} / N_{A}\right)$ vs. $n_{\varphi}$ 
$R_{y}=\frac{\omega \mu_{0}}{2 \pi N_{a}} \int_{-\infty}^{+\infty} g\left(k_{z}\right)\left(\frac{\left|J_{a . y}\left(k_{x}\right)\right|}{I_{a .0}}\right)^{2} d k_{z}$ and

$X_{y}=\frac{\omega \mu_{0}}{2 \pi N_{a}} \int_{-\infty}^{+\infty} h\left(k_{\varphi}\right)\left(\frac{\left|J_{a . y}\left(k_{z}\right)\right|}{I_{a .0}}\right)^{2} d k_{z}(\Omega / \mathrm{m})$.

$R_{y}$ and $X_{y}$ are plotted in Fig. 3 as function of (integer values) of $n_{\varphi}$.

\section{FW Current Drive}

As noted above, we shall assume an operation at a sufficiently low frequency $f_{e}$ (in DEMO $f_{e} \approx 25 \mathrm{MHz}$ ) so as to exclude from the plasma cross section all ion and fusion-born $\alpha$-particle resonances and to let electron damping emerge. The active power of a FW launched at $\theta_{\varphi}=\pi / 2$ and $f<f_{e}$ is then entirely dissipated on electrons by non-collisional damping and can generate an electron convection current density due to the asymmetry of the power spectrum. The amount of power transferred depends on the E\&K parameter $w\left(\varrho, n_{\varphi},\right)$ which, in turn, depends on the electron temperature profile $T_{e}$. We shall use DEMO - like electron $T_{e}$ and $n_{e}$ profiles as [8]:

$$
\begin{aligned}
& T_{e}(\rho)=\left(T_{e .0}-T_{e .1}\right)\left(1-\rho^{2}\right)^{\alpha_{T}} \text { and } \\
& n_{e}(\rho)=\left(n_{e .0}-n_{e .1}\right)\left(1-\rho^{2}\right)^{\alpha_{n}}
\end{aligned}
$$

$\rho=r / a$, is the normalized radial coordinate such as $\mathrm{R}(\rho)=\mathrm{R}_{0}+\mathrm{a} \rho$. The E\&K dimensionless formula for radio frequency (RF) $\mathrm{CD}$ efficiency $\tilde{\eta}(\epsilon, \vartheta, w)$ in presence of magnetic trapping [3] is a function of $i)$ the inverse aspect ratio of the flux surfaces $(\epsilon)$, ii) the poloidal location where the RF power is dissipated $(\vartheta)$ and iii) the ratio of toroidal phase velocity and electrons thermal speed $w=\frac{v_{p h . \varphi}}{v_{t h e}}$, called in the following $\mathrm{E} \& \mathrm{~K}$ parameter.

From a power flow point of view a RA can be regarded as round conductor of radius $R_{a}$ of complex impedance p.u.l. $Z_{A}$ carrying a surface current $I_{A}$. The Poynting vector $\boldsymbol{P}=(\boldsymbol{E} \times \boldsymbol{H}) \approx-E_{y} H_{\varphi}$ is almost everywhere radial and directed toward the torus axis. The power density p.u.l. through the cylindrical surface at $\mathrm{R} \approx \mathrm{R}_{0}+\mathrm{a}_{0}$ and absorbed by plasma according to the Pointing theorem is:

$$
\int_{V} P_{e} d V=\int_{V} \operatorname{Re}(\boldsymbol{P}) d V=\operatorname{Re}\left(Z_{A}\right) I_{A}^{2} \approx \int_{V} f_{P} d V(6)
$$

with a radial distribution depending on $\mathrm{E} \& \mathrm{~K}$ parameter $w\left(\rho, T_{e}\right)$ as $f_{P}\left(\rho, T_{e}\right) \approx w\left(\rho, T_{e}\right) e^{-\frac{w\left(\rho, T_{e}\right)}{2}}$

which is maximum for $w=1$ and rapidly decreases for $w>1$. As function of $\rho=\frac{R-R_{0}}{a}$ and $n_{\varphi}$

$$
w\left(\rho, n_{\varphi}\right)=\frac{v_{p h . \varphi}}{v_{t h . e}}=\frac{1}{n_{\varphi}} \frac{2 \pi R_{a} f}{c} \sqrt{\frac{m_{e} c^{2}}{T_{e}(\rho)}} .
$$

$f_{P}\left(\rho, T_{e}\right)$, is plotted in Fig 4 vs. $\rho$ and $n_{\varphi}$. As seen, it tends to increase with $n_{\varphi}$ because of the increasing loading $\mathrm{R}$ (and therefore of the power available to the electrons). However, the power is mainly deposited in the plasma core at low $n_{\varphi}$ whereas it disperses to the plasma edges at high $n_{\varphi}$.

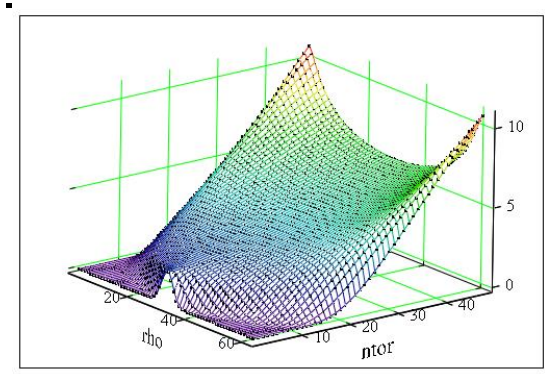

Fig $4 f_{P}\left(\rho, n_{\omega}\right)$

Only the fraction $f_{C D}$ contributes to $\mathrm{CD}$ because of toroidal trapping, a condition only depenent on the ratio $v_{e \|} / v_{e \perp}$. The circulating electrons are those within the loss cone $\theta_{S}=\arcsin \sqrt{\frac{1}{R_{S}(\rho)}}$. where the mirror ratio $R_{S}(\rho)=\frac{B_{\max }(\rho)}{B_{\min }(\rho)} \approx \frac{R_{0}+r}{\left.R_{0}-r\right)}$ and, for a poloidal angle $(\vartheta=0)$ launch, $r=a_{0} \rho$. The power fraction $f_{C D}$ dissipated on circulating electrons (and obtained by setting $P_{A}$ to zero for $\rho>R_{S}(\rho)$ ) is plotted in Fig. 5.

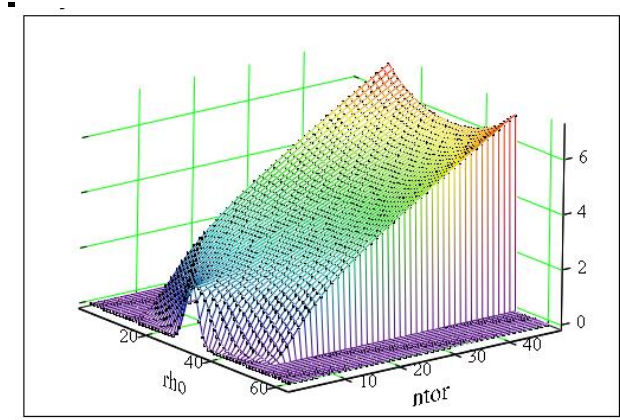

Fig. $5 f_{C D}\left(\rho, n_{\varphi}\right)$

Fig 5 shows that at low frequency magnetic trapping significantly reduces $($ by $\approx 50 \%$ ) the power available for CD at high $n_{\varphi}$ values, in qualitative agreement with [7]. The power density p.u.l. $P_{e}$ available for CD can be estimated from (6) as:

$$
2 \pi \int_{-\rho}^{\rho} P_{C D}\left(\rho, n_{\varphi}\right) f_{C D} \rho^{\prime} d \rho^{\prime}=\operatorname{Re}\left(Z_{A}\left(n_{\varphi}\right) I_{A}^{2}\right.
$$

and the CD driven in the plasma p.u.l can be estimated from [5] as:

$$
J_{C D}\left(\rho, n_{\varphi}\right)=\frac{0.38}{\ln \lambda} \frac{T_{e}(\mathrm{keV})}{n_{e .20}} P_{C D}\left(\rho, n_{\varphi}\right) \tilde{\eta}\left(\rho, n_{\varphi}\right)
$$

where $\tilde{\eta}\left(\rho, n_{\varphi}\right)$ is the dimensionless E\&K CD efficiency computed for a LF equatorial launch $(\vartheta=0)$ which can be approximately fitted, by :

$$
\tilde{\eta}\left(\rho, n_{\varphi}\right)=17.22 w\left(\rho, n_{\varphi}\right)^{0.22}-13.36
$$

plotted in Fig. 6 for $0.3<w<3$, and $Z_{\text {eff }}=1$. $J_{C D}\left(\rho, n_{\varphi}\right)\left(\mathrm{A} / \mathrm{m}^{3}\right)$ is plotted in Fig 7 for an on-axis $T_{e}(0)=39.5 \mathrm{keV}$, a phase advance $\theta_{\varphi}=\frac{\pi}{2}, f \approx 20$ $\mathrm{MHz}$ and $I_{A}=1 \mathrm{~A}$. 


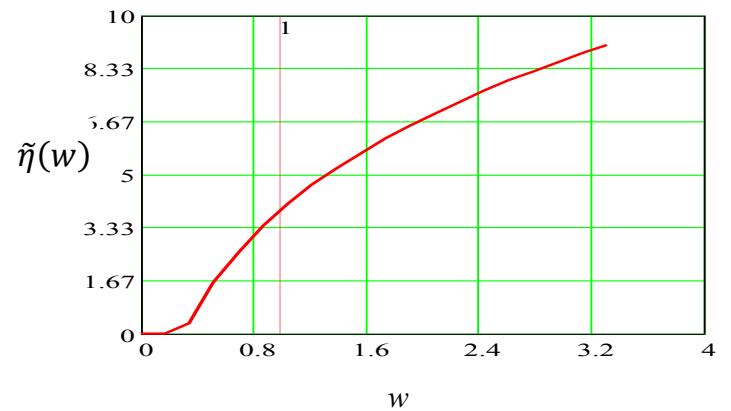

Fig. $6 \tilde{\eta}\left(w, \vartheta=0 Z_{\text {eff }}=1\right)$

The best on-axis CD efficiency is obtained for $\mathrm{w}\left(0, n_{\varphi}\right)=1$, or for an optimum $n_{\varphi}$ :

$$
\widehat{n_{\varphi}}=\frac{2 \pi R_{a} f}{c} \sqrt{\frac{m_{e} c^{2}}{T_{e}(R)}} \approx 10
$$

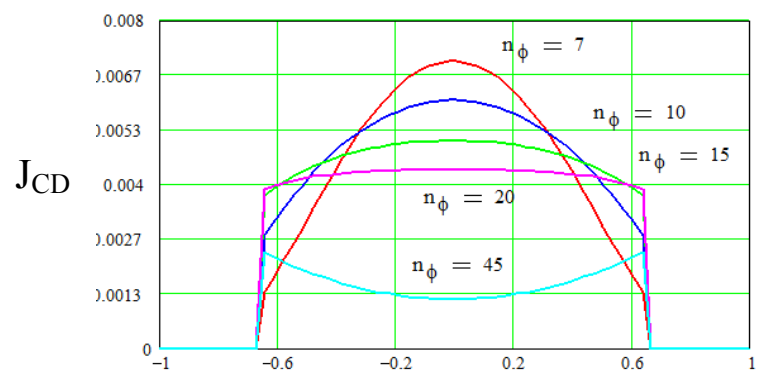

Fig $7 J_{C D}\left(\rho, n_{\varphi}\right)\left(\mathrm{A} / \mathrm{m}^{3}\right)$

i.e. for a number of elements $\widehat{N_{a}}=4 \widehat{n_{\varphi}}=40$. For onaxis $T_{e}(0)=39.5 \mathrm{keV}, \quad n_{e .20}=1$ a phase advance $\theta_{\varphi}=\frac{\pi}{2}$ and $f \approx 20 \mathrm{MHz}$ an integrated CD efficiency $\eta_{C D} \approx 130 \mathrm{kA} / \mathrm{MW}$ results (Fig. 8).

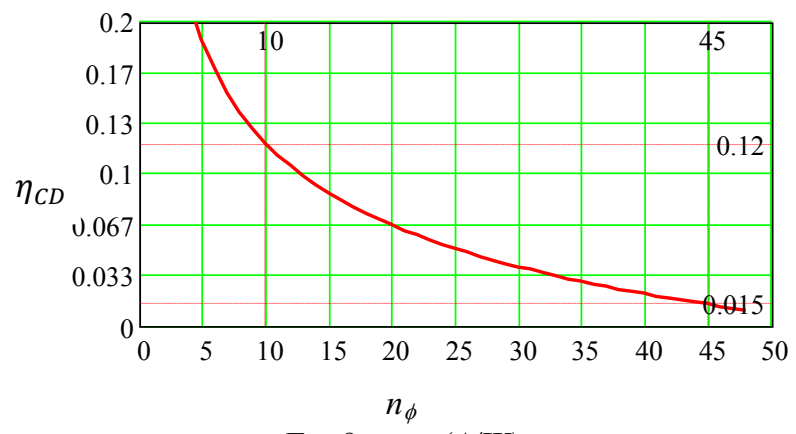

Fig 8. $\eta_{C D}(A / W)$

\section{Conclusions}

In this paper, the CD operation of a Ring Array FW launcher introduced in [9] is dicussed using a simple model. The RA appears to have a number of advantages compared to existing and proposed in-port ICRF launchers operating at high $n_{\phi}$ :

- Low frequency operation ( $\mathrm{f}<25 \mathrm{MHz}$ in DEMO) avoids competition with much more efficient ion and fusion born $\alpha$-particle absorption .
- High E-fields usually resulting from the FW evanescence and causing undesiderably high $\mathrm{AC}$ and DC potential at the plasma edge can be reduced to a safer level.

- The proposed individual and independent real time control of phase and magnitude of the RA currents (associated with an automatic matching by an overall frequency control) allows real time regulations of the power spectrum that could be used to improve stability of the drive in variable plasma conditions.

- The best CD performances of the RA low frequency operation are obtained at values of $n_{\varphi}(\approx 10)$. For an array phase advance $\theta_{\varphi}=\frac{\pi}{2}$, (maximum momentum per power expenditure) the array number of elements is $N_{a} \approx 40$.

- A full metal construction of the in-vessel components and an acceptable integration in a modular blanket appears possible.

- Although not discussed here, bulk plasma heating and ion minority $\mathrm{CD}$ could also possible, the latter used for reactor particles exaust . H and CD mode(s) of operation could be used in conjunction in bringing the reactor to full operation.

- The present analysis shows a CD efficiency higher than that predicted by present estimates for a ITERlike types of launchers. It also shows that the results depend considerably on plasma parameters at present not easily predictable in a commercial reactor. Critical plasma parameters are low electron damping (not necessarily described by single pass absorption) and the control of the electron temperature profile, affected by the power dissipated at the edge by trapped electrons.

- In view of the significant difference between the RA and ITER like arrays so far investigated, an analysis including more refined plasma descriptions is planned

\section{References}

[1] H. Zohm et al, Proc. EPS Conf. on Plasma Phys. ECA37 DO3, 108 (2013)

[2] G.Federici et al, Fus. Eng. and Design 89 (2014) 882-889

[3] D.Ehst, C. Karney (1991) Nucl. Fusion 311933

[4] R. Bilato et.al, J.of Phys.Conf. Series 561, (2014).

[5] E. Lerche, et al. AIP Conf. Proc. 1580, 338 (2013).

[6] M.Porkolab et al, Plasma Phys. Cont. Fus. 40 A35 (1998)

[7] Ye.Kazakov et al, Plasma Phys and Cont.Fus. 57,(2015), 0.25014.

[8 M.Brambilla et al, Nuclear Fusion (2015) 55(2) :023016

[9] G.Bosia, Fus.Eng. and Design.92(2015) 8-15

[10]A.M.Messiaen et al. Proc. 3rd Joint V-G Int. Symp. on Fus.Tech.1,675 (1984)

[11] G.Bosia "Heating and Current Drive in a Ring Array", Paper to be submitted for publication 